\title{
Estrategias Pedagógicas Virtuales para la enseñanza - aprendizaje en la Educación Superior
}

\author{
Mariela Svetlichich Duque
}

Facultad de Ciencias Económicas y Administración, Universidad de la República,

Uruguay.

\begin{abstract}
Resumen
El presente artículo describe la evolución de la educación virtual universitaria en los tiempos actuales. En primer lugar, se exponen las características de los nuevos contextos de enseñanza y la importancia de la formación en competencias, y se realiza una revisión de las habilidades que deben desarrollar los estudiantes para su desempeño en el siglo XXI, propuesta desde diversos ámbitos académicos. Luego, se analiza la incorporación de las TIC en la universidad, así como de la innovación y la utilización de la tecnología en el aula mediante la identificación de diversas estrategias para ello. En esta línea, se precisa cómo seleccionar el enfoque metodológico de enseñanza - aprendizaje, desde los diferentes modelos pedagógicos de la educación en general y a través del análisis de diferentes teorías de enseñanza. Posteriormente, se considera la contextualización de los aprendizajes, la utilización de la plataforma educativa y de diversas líneas de actuación para la incorporación de las TIC, así como se realiza el desarrollo de un curso con las características de las diferentes actividades que deben ser desarrolladas. En relación a ello, se presentan los recursos para la elaboración de los materiales a utilizarse en un curso acorde con las características de los alumnos. Por último, se describen los diferentes roles docentes y las competencias que deben ser desarrolladas según cada función.
\end{abstract}

Palabras Clave: tecnología, competencias, aula virtual, educación virtual

\section{Virtual Pedagogical strategies for teaching - lear- ning in higher education}

\begin{abstract}
The work connects us with the evolution of the university Virtual Education in the present times. At the beginning the characteristics of the new teaching contexts are exposed, and the importance of the training in competitions. A review is made of the
\end{abstract}


skills that students should develop for their performance in the 21st century, proposed by various academic fields then analyzed the incorporation of ICT in the university, innovation and the use of technology in the classroom, as well as the analysis of various strategies for this. It analyses how to select the methodological approach of teaching-learning, from the different pedagogical models of the education in general, analyzing different theories of teaching. It is considered the contextualization of the learning, the utilization of the educational platform and various lines of action for the incorporation of the TICs is carried out the development of a course with the characteristics of the different activities that must be developed. Resources are presented for the elaboration of the materials to be used in a course according to the characteristics of the students. Finally, we describe the different teaching roles and the competencies that must be developed in each one of them.

Keywords: technology, competencies, virtual classroom

\title{
Estratégias pedagógicas virtuais para o ensi- no-aprendizagem no ensino superior
}

\begin{abstract}
Resumo
O trabalho nos conecta com a evolução de educação virtual na Universidade nos tempos atuais. As características dos novos contextos de ensino são expostas, e a importância do treinamento em competições. Uma revisão é feita das habilidades que os estudantes devem desenvolver para seu desempenho no século XXI, propor por vários campos académicos analisou então a incorporação das TIC na Universidade, na inovação e no uso da tecnologia Na sala de aula, bem como a análise de várias estratégias para isso. Analisa como selecionar a abordagem metodológica do ensino-aprendizagem, a partir dos diferentes modelos pedagógicos da educação em geral, analisando as diferentes teorias do ensino. Considera-se a contextualização da aprendizagem, a utilização da plataforma educacional e várias linhas de ação para a incorporação do TICs é realizado o desenvolvimento de um curso com as características das diferentes atividades que devem ser Desenvolvido. Os recursos são apresentados para a elaboração dos materiais a serem utilizados em um curso de acordo com as características dos alunos. Por fim, descrevemos os diferentes papéis didáticos e as competências que devem ser desenvolvidas em cada uma delas.
\end{abstract}

Palavras-chave: tecnologia, competências, sala de aula virtual 


\section{Introducción}

Los cambios tecnológicos son cada vez más vertiginosos y, a su vez, las transmisiones de saberes y prácticas entre generaciones están cambiando de forma acelerada e incierta. En este sentido, como menciona Keri Facer (Facer, 2011), para el 2030, no solo se extenderá el ciclo vital y predominará una pirámide poblacional cada vez más alta, sino también continuará el crecimiento exponencial del acceso masivo a la tecnología. En consecuencia, tras abaratarse los costos y precios de los aparatos tecnológicos, se incrementará la sinergia entre tecnologías y seres humanos, por lo que artefactos cada vez más pequeños serán empleados con mayor frecuencia a través de una red digital infinitesimal.

En líneas generales, es posible señalar que el siglo XXI ha llegado como un símbolo de cambio permanente, del desarrollo de las telecomunicaciones que acortan distancias; sin embargo, también ha dejado espacio a un concepto relegado durante siglos por la ciencia y por la educación: la dimensión emocional del ser humano. Como dice S. De La Torre, son signos del tiempo actual: la tecnología, la emoción y el cambio. Así, tecnología y humanismo pasan a ser conceptos complementarios.

Considerando este contexto, el aprendizaje para toda la vida, la comprensión de contextos y situaciones que exigen la toma de decisiones argumentadas, y las posibilidades de análisis y de crítica ante diversos enunciados se han identificado como competencias que deben ser fuertemente desarrolladas ante el cambiante estado de las Tecnologías de la Información y la Comunicación (TIC), y el vertiginoso avance de los conocimientos sobre aquellos aspectos que demanda una sociedad de futuros profesionales universitarios (Svetlichich, 2015).

\section{Marco Teórico}

\subsection{Las habilidades para el Siglo XXI}

El currículo de la era industrial, con su énfasis en la transmisión y reproducción de conocimientos, no está siendo capaz de preparar a los estudiantes para vivir y desenvolverse con éxito en la era globalizada del conocimiento y afrontar un mundo que está en continua transformación. Bajo esta premisa, cada vez se reconoce más la necesidad de una formación que sea pertinente para la sociedad; por ello, la formulación de competencias genéricas, que derivan en otras más especializadas, constituye el horizonte de acciones de formación deseables en educación superior $y$, a la vez, son un referente de gran importancia para poder monitorear la calidad de la formación en todos los programas académicos. La apuesta por competencias genéricas que sean transversales a todos los niveles educativos, y a los diferentes énfasis y programas de formación es, entonces, una respuesta a las necesidades de la sociedad actual. 
Actualmente, se destaca que la metodología constituye la herramienta o palanca esencial para llevar a cabo un cambio educativo desde los tradicionales contenidos académicos hacia contenidos profesionales centrados en el desarrollo de competencias y habilidades. No obstante, la propuesta de nuevas metodologías educativas debe considerar, entre otros aspectos, que los jóvenes que nacieron en la cultura digital han forjado una generación excesivamente expuesta a consumos digitales, lo que permite que ejerciten sus capacidades cognitivas, mucho más que las generaciones que les precedieron, expuestos a la cultura escrita (Johnson S. , 2005).

Atendiendo a esta idea, a continuación, se analizan algunas iniciativas internacionales, las cuales intentan generar un mapa de habilidades del siglo XXI. Algunos ejemplos destacados son los siguientes:

\subsubsection{Iniciativa ATC21S (Universidad de Melbourne)}

Desde el 2009, la iniciativa ATC21S, con sede en la Universidad de Melbourne y auspiciada por Intel, Cisco y Microsoft, entiende como "competencias del siglo XXI" a las destrezas, conocimientos y actitudes necesarias para enfrentar exitosamente los retos de esta época, que invitan a reformular las aspiraciones en materia de aprendizaje (Competencias del siglo XXI, 2017). Asimismo, ha indicado 10 habilidades centrales que se asientan en cuatro grandes ejes. Son los siguientes:

- Maneras de pensar (3): creatividad e innovación, pensamiento crítico, resolución de problemas y aprender a aprender

- Herramientas para trabajar (2): apropiación de las tecnologías digitales, manejo de la información y capacidad de evaluarla en forma crítica

- Maneras de trabajar (2): comunicación, y colaboración y trabajo en equipo

- Maneras de vivir en el mundo (3): capacidades de planeamiento y fijación de metas; capacidad de tomar decisiones y actuar en consecuencia con ellas; capacidad de asumir un rol activo, reflexivo y constructivo en la comunidad local, nacional y global

\subsubsection{Institute for the Future (Universidad de Phoenix)}

Considerando la relación de la educación con el mundo laboral, el Institute for the Future de la Universidad de Phoenix postula 10 habilidades laborales fundamentales para el futuro:

- Capacidad para crear sentido: habilidad para comprender el significado real de lo que se está expresando

- Inteligencia social: habilidad para conectarse con otros de una forma compleja y directa mediante la estimulación de interacciones potentes

- Pensamiento nuevo y adaptativo: capacidad para crear soluciones y respuestas más allá de la norma 
- Competencias multiculturales: habilidades para operar en diferentes escenarios culturales

- Pensamiento computacional: habilidad para traducir grandes cantidades de datos en conceptos abstractos y capacidad para entender razonamientos basados en datos

- Alfabetización en nuevos medios: habilidad para evaluar críticamente y crear contenidos a través de los nuevos medios con el objetivo de una comunicación persuasiva

- Pensamiento transdisciplinario: capacidad para comprender y usar conceptos de distintas disciplinas

- Capacidad de diseño: habilidad para representar y desarrollar tareas y procesos con el objetivo de lograr los resultados deseados

- Gestión de cargas cognitivas: habilidad para filtrar y seleccionar información según su importancia, comprendiendo cómo maximizar sus usos cognitivos mediante una diversidad de herramientas

- Colaboración virtual: habilidad para trabajar productivamente a través del desarrollo del compromiso y en equipo

\subsubsection{OCDE - Organización para la Cooperación y el Desarrollo Económi- Co}

Por su parte, la OCDE señala tres categorías de competencias centrales (OCDE, 2010):

- Uso de herramientas interactivas: lenguaje, textos, conocimientos, tecnologías

- Interactuar en grupos heterogéneos: capacidades de relacionamiento, cooperación, trabajo en equipo y resolución de conflictos

- Actuar autónomamente: contar con proyectos de vida, y establecer y defender intereses, necesidades, límites y derechos

\subsubsection{Otros autores}

El Prof. Howard Gardner de la Universidad de Harvard analizó que las mentes deben ser desarrolladas para desempeñarse en un mundo globalizado. En este sentido, es responsabilidad del docente desarrollarla en sí mismo para, luego, promover su desarrollo en los estudiantes.

Según este autor, por un lado, existen tres mentes cognitivas, que se refieren al trabajo usual en educación (Gardner, 2005): la mente disciplinada, que domina conocimientos y habilidades; la mente sintética, que decide qué debe ser atendido para una investigación con detalle y qué debe ser ignorado; y la mente creativa, que se aventura por territorios inexplorados. Para todos los casos, el conocimiento disci- 
plinado es necesario para sintetizar y desarrollar nuevas ideas, conceptos, historias, teorías y habilidades, en la medida en que busca demostrar que estas siguen siendo deseables e indispensables.

Por otro lado, también existen dos tipos de mente que se relacionan al tratamiento de la esfera humana: la mente respetuosa, que valora y estima la diversidad, y trata de trabajar de manera efectiva con individuos de todos los orígenes y procedencias, respetando las capacidades de cada uno; y la mente ética, que procede sobre la base de principios, buscando actuar en función de una sociedad más amplia. Esto conduce a repensar la enseñanza y las características de los actores involucrados en ella (Svetlichich, Educación a distancia: Competencias docentes para alumnos interactivos, 2013).

Finalmente, en relación al corte de las habilidades necesarias para la vida digital, en un marco de interacción permanente con las nuevas tecnologías, Henry Jenkins postula que la alfabetización digital con nuevos medios debe integrar los siguientes factores: el juego, la simulación, la performance, el multitasking, la cognición distribuida, la inteligencia colectiva, la capacidad de juicio, la navegación transmedia, la colaboración y la negociación (Jenkins, 2006). Estos saberes y habilidades reformulan las bases del currículo y las didácticas en la educación superior, dado que enseñar estas competencias requiere de docentes que las perciban como propias.

\subsection{Las TIC en la docencia Universitaria}

Como se puede inferir del apartado previo, la enseñanza universitaria está actualmente en un momento de transformación y búsqueda de un nuevo sentido del conocimiento comprometido por la realidad social y la demanda de calidad. En este sentido, las instituciones de enseñanza, atendiendo a estos cambios sociales, económicos y tecnológicos, se encuentran adaptando los procesos educativos a las nuevas circunstancias.

En un contexto donde se requiere que los estudiantes adquieran competencias para las cuales el sistema educativo no fue concebido, las TIC pueden contribuir para saldar la diferencia (Falco, 2017). Así, por ejemplo, la Conferencia Mundial de Educación Superior realizada en París y organizada por la UNESCO permitió describir las nuevas dinámicas educativas, así como reconocer el potencial de las TIC como una herramienta que debe utilizarse para la renovación de la educación superior, amplificando y diversificando la trasmisión del saber, y posicionando los conocimientos y la información a disposición de un público más amplio (UNESCO, Aprendizaje movil para docentes en América Latina. Análisis del potencial de las tecnologías móviles para apoyar a los docentes y mejorar sus prácticas, 2012).

Esto invita a pensar en innovaciones educativas que configuran un nuevo contexto donde la presencia de las telecomunicaciones y la necesidad de formar profesionales para tiempos de cambio exigen nuevos procesos de enseñanza - aprendizaje, así como nuevos modelos que se adapten a ellos. En este sentido, el aula virtual es considerada una innovación en la inserción de las TIC; no obstante, la experiencia 
y la literatura demuestran que, usualmente, se transfiere un modelo tradicional de formación al espacio virtual, que apela a metodologías conductistas. Así, se cambia del aula presencial al aula virtual, pero se recrean los mismos funcionamientos (AdeII, 2004).

Bajo esta premisa, cabe la pregunta de por qué triunfaron, en su momento, el pizarrón, el cuaderno de clases y el libro de texto. Se puede decir que estas tecnologías se alinearon con ciertas necesidades del sistema educativo: el método expositivo como núcleo de la enseñanza en el aula (el pizarrón), la realización de ejercicios que requería visibilizar y evaluar el trabajo continuo de los alumnos (el cuaderno), y la necesidad de masificar los contenidos curriculares a través de la implementación de formatos comprensibles y de uso diario (el libro de texto).

Ahora bien, ¿qué sucede con las herramientas tecnológicas? Diversos estudios confirman que las TIC están presentes en la docencia universitaria, pero ligadas, la mayoría de las veces, a usos tradicionales y con un impacto positivo, pero superficial en el aprendizaje, dado que las tecnologías más usadas son aquellas que los docentes emplean en otros contextos de su desarrollo profesional y no aquellas que serían más provechosas para el aprendizaje del alumnado. En específico, si se analizaran los programas de Contabilidad, se encontraría que muy pocas universidades, por no decir casi ninguna, utiliza un programa de Contabilidad para enseñar a registrar, pese a que, en la vida profesional, ya no existen contabilidades manuales. En este sentido, como menciona N. Peré, la innovación pedagógica y la tecnológica son dos procesos diferentes que pueden darse juntos, pero no necesariamente de forma articulada (Peré, 2017).

Por otra parte, en términos de Peré y Rodés, "la mera incorporación de tecnología en los procesos educativos no garantiza la innovación ni el cambio de las formas de enseñar, aprender y vincularse entre los diferentes actores del hecho educativo, transformación que sí está vinculada a la metodología de enseñanza y de aprendizaje que se proponga" (Rodes \& Peré, 2005). Considerando esta idea, es posible señalar que, en muchas ocasiones, se enseña, y se desconoce la razón y los propósitos de dicha enseñanza; en otras palabras, no se logra identificar con claridad qué es lo que se busca en el estudiante cuando se abordan unas determinadas temáticas ni por qué este debe asimilarlas. Por consiguiente, muchos de los esfuerzos de los docentes no se traducen en un aprendizaje significativo para los estudiantes, lo que implica un olvido rápido de estos contenidos y habilidades.

Esto lleva a precisar dos preguntas: para qué los estudiantes aprenden y para qué los docentes enseñan. La primera responde a generar en el estudiante una mayor autonomía, focalizando el aprendizaje para lograr una conexión significativa con el saber, que le permita un desempeño en el mundo laboral. En este sentido, se puede afirmar que, entre más claridad posea el estudiante en sus propósitos de formación, más autónomo será, en la medida en que sabrá hacia dónde debe ir y dirigirá todos sus esfuerzos cognitivos al logro de dichas metas. 
Por otro lado, cuando se responde a la segunda pregunta desde la docencia, se busca generar un escenario coherente que permita al estudiante adquirir la competencia enseñada. Del mismo modo, identificar los propósitos de formación orienta en la generación de escenarios de aprendizajes más coherentes con los niveles de competencia propuestos, así como a dirigir un proceso de asesoramiento de mayor calidad.

Dentro de un contexto de educación virtual, contar con una respuesta clara a estas preguntas es de vital importancia para los dos principales actores del proceso de enseñanza - aprendizaje: el docente y el estudiante, respectivamente.

\section{Resultados de la Investigación}

\subsection{Estrategias para la incorporación de las TIC en el aula}

La UNESCO recomienda que la formación universitaria incluya a las TIC para poder garantizar la adquisición de las competencias; contribuir en la búsqueda y en la evaluación de la información; ayudar en la toma de decisiones; mejorar la comunicación y la interacción, la difusión del conocimiento y, en definitiva, la productividad de todos los profesionales (UNESCO, 2008). Es difícil imaginar una universidad actual que pueda ofrecer todo esto sin el concurso eficaz de las TIC. En la formación universitaria, esto supone un reto ineludible para las instituciones, que está directamente asociado con la formación de los docentes, no siempre preparados en esos temas. Por ello, a continuación, se analiza qué elementos considerar para la introducción de las TIC en el aula.

\subsubsection{Seleccionar el enfoque metodol ógico de enseñanza - aprendizaje}

El aprendizaje es un proceso de construcción individual y social, que el estudiante debe regular, en tanto es responsable del mismo, pero ¿cómo lograr un aprendizaje independiente y autónomo? Enfrentándolos a situaciones en las que deban utilizar estrategias de búsqueda de información, aplicar los nuevos conocimientos para la solución de problemas realistas, tomar decisiones y trabajar en forma autónoma, reflexiva y crítica son algunas de las respuestas posibles.

Cuando se atiende al propósito de la formación, se observa que el énfasis ha pasado de la enseñanza al aprendizaje. Esto es válido para cualquier tipo de enseñanza, de presencial a virtual, de primaria a la universidad. De este modo, es interesante ver el modelo pedagógico utilizado y analizar cómo se interrelacionan los tres polos de la tríada didáctica: docente/alumno/conocimiento o saber mediante los procesos que están implícitos en el acto educativo (Svetlichich Duque, 2011).

Por un lado, los modelos centrados en la enseñanza privilegian el eje profesor - saber, en el que el docente conoce la disciplina a enseñar, mientras que el estudiante, que no maneja un conocimiento, aprende. En este sentido, bajo este eje, el proceso de aprendizaje se realiza dentro de una secuencia lógica y se adquiere por recep- 
ción. Asimismo, se promueve la teoría sobre la práctica. Es posible señalar que la mayor parte de los docentes universitarios es representada mediante estas características y que este modelo prevalece en la mayoría de las universidades, incluso en programas de educación a distancia.

Por otro lado, los modelos centrados en el aprendizaje privilegian el eje alumno saber, dado que se piensa la docencia desde la perspectiva del que aprende, no del que enseña. En este modelo, el alumno es el elemento activo y el docente solo constituye un factor facilitador. Asimismo, el aprendizaje implica una modificación en los esquemas referenciales y conductuales del sujeto, que, tras considerar un enfoque "constructivista", también ha modificado y mejorado las prácticas docentes. Dicho de otro modo, es un proceso dinámico en el que lo más importante es aprender a aprender. Es posible mencionar que la mayoría de los programas de educación a distancia están pensados desde este modelo.

Por último, los modelos centrados en la formación destacan las relaciones pedagógicas, (alumno - alumno, alumno - docente, docente - docente) como motivadores de una dinámica de desarrollo personal. Parafraseando a P. Freire, se podría decir que "nadie forma a nadie, pero tampoco nadie se forma solo; los hombres se forman en relación mutua".

Ahora bien, con la introducción de la tecnología en la enseñanza, aparece un nuevo paradigma en la enseñanza - aprendizaje, que implica conceptos como los de "colaboración" y "enseñanza asincrónica", que conducen a una revolución pedagógica donde las viejas estructuras inmóviles de espacio - tiempo pierden su sentido (Lopez Segrera). En la actualidad, diversas líneas de investigación consideran que las nuevas formas de enseñanza - aprendizaje requieren de espacios distintos a los tradicionales para llevar a cabo dichos procesos.

En esta línea, muchas universidades han apostado, en los últimos tiempos, por metodologías activas de enseñanza - aprendizaje, como la "Clase invertida", donde se utilizan herramientas que facilitan las interacciones mediante la atención en la movilidad y la múltiple utilización de los dispositivos tecnológicos. Dicho de otro modo, estas metodologías activas son métodos, estrategias y técnicas que el docente emplea para transformar el proceso de enseñanza en una serie de actividades que, mediante la participación activa del estudiante, lo conduzcan al aprendizaje (Labrador \& Andreu, 2008). En otras palabras, se cambia el foco de los contenidos y se pasa a actividades, que centran el proceso formativo en el estudiante y que responden de mejor forma a los estilos de aprendizaje que presentan los estudiantes a la hora de enfrentarse a las tareas educativas (Bolivar \& Rojas , 2014).

Finalmente, la integración de espacios de aprendizajes físicos y virtuales, en un mismo curso, ha introducido una forma diferente de concebir el aprendizaje híbrido o blendlearning, el cual utiliza un entorno virtual flexible, que permite incorporar funcionalidades para que el aprendizaje sea más social para los estudiantes. Este se realiza a través de diversos canales de comunicación a distancia, presencial, asincrónica y sincrónica, donde la participación de los estudiantes, desde diferentes lugares, se convierte en un beneficio clave (Higher, 2015). 


\subsubsection{Los aprendizajes contextualizados}

Los aprendizajes contextualizados buscan otorgar sentido a los contenidos $y$, por ello, se afirma que "se aprende solo aquello que se aplica y que es útil para la vida". El docente posee el compromiso de diseñar contenidos con elevados niveles de aplicación práctica, de tal forma que estos cobren relevancia en la formación de los estudiantes. Con este objetivo, es importante que los contenidos sean problematizados y que, para su entendimiento, requieran de una búsqueda de soluciones por parte de los estudiantes. De acuerdo con Coll, Pozo, Sarabia y Valls (Coll, Pozo, Sarabia, \& Valls, 1992), los contenidos que se enseñan se pueden agrupar en tres áreas básicas: conocimiento declarativo, procedimental y actitudinal. Atender a esta clasificación implica especificar los contenidos bajo estas tres categorías para crear conciencia sobre lo que se enseña, sin desconocer que estos conocimientos son complementarios y que ninguno es más importante que otro (Diaz \& Hernández, 2002). Estos pueden observarse en el siguiente cuadro:

\section{Cuadro 1. Tipos de saberes}

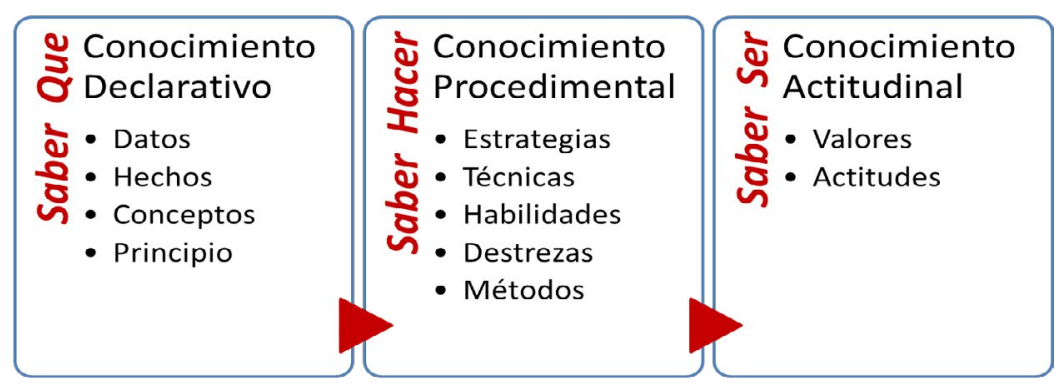

Fuente: Elaboración propia a partir de Coll, Pozo, Sarabia y Valls, 1992

Asimismo, uno de los objetivos perseguidos por la educación universitaria es dotar a los estudiantes de habilidades necesarias para el ámbito laboral. En esta línea, Wickline define al aprendizaje profundo como el dominio de los contenidos que involucra el pensamiento crítico de los estudiantes, la resolución de problemas, y al aprendizaje autónomo y colaborativo (Wickline, 2014). Con el fin de mantener la motivación en el aprendizaje, los estudiantes deben ser, entonces, capaces de establecer conexiones claras entre el plan de estudios y el mundo real, así como comprender que los nuevos conocimientos y las habilidades influirán sobre ellos (Gonring, P; Ramsey, B, 2016).

Por último, el aprendizaje basado en proyectos o desafíos fomenta experiencias de aprendizaje más activas tanto dentro como fuera del aula. Bajo este modelo, basándose en una pregunta central o en un concepto, el estudiante deberá llevar a cabo la investigación de los objetivos definidos, lo que favorece la construcción de conocimiento significativo.

\subsubsection{La plataforma educativa}

Un entorno virtual de aprendizaje (EVA), ambiente virtual de aprendizaje (AVA) o Vir- 
tual Learning Environment (VLE) es una aplicación informática, diseñada para facilitar la comunicación pedagógica, mediada por la tecnología, entre los participantes de un proceso educativo, sea este completamente a distancia o de una naturaleza mixta, que combine ambas modalidades en diversas proporciones (Silva Quiroz, 2004). En un entorno virtual de aprendizaje, se encuentran espacios para las siguientes funciones: distribuir materiales educativos en formato digital (textos, imágenes, audio simulaciones, juegos, etc.), realizar discusiones en línea, integrar contenidos relevantes de la red, y permitir participaciones de expertos o profesionales en debates y charlas. Asimismo, se combinan herramientas diversas para la comunicación sincrónica y asincrónica, la gestión de los materiales de aprendizaje y de los estudiantes, los sistemas de evaluación y seguimiento de los procesos educativos, y el soporte tecnológico a profesores y estudiantes que permitan optimizar el proceso de enseñanza- aprendizaje (García Aretio, Ruiz , \& Dominguez, 2007).

Si bien la implementación de un EVA no garantiza la innovación ni la mejora de la calidad de la enseñanza, es un comienzo para la incorporación de las TIC en el aula (Adell, 2004). En esta medida, diseñar e implementar un EVA para innovar en las prácticas formativas virtuales requiere prestar atención a tres áreas fundamentales: a los planes de formación de los docentes, que consideren competencias digitales; a desarrollar metodologías de enseñanza - aprendizaje centrados en el estudiante; $y$ al rol del docente como diseñador y moderador del EVA.

\subsubsection{Líneas de actuación para la incorporación de las TIC}

Existe una serie de líneas de acción basadas en diferentes técnicas asociadas a la incorporación de las TIC, las cuales se relacionan a metodologías de aprendizaje centradas en el estudiante que construye su propio conocimiento, y en el marco de una estrategia liderada por el docente que elabora y diseña el curso. El planteamiento de la educación centrada en el estudiante ha incitado a muchos actores educativos a replantearse la forma en que deben ser configurados los espacios de aprendizaje. En este sentido, cada vez más se investiga el diseño ideal del espacio educativo, en la medida en que la universidad se aleja de la programación basada en la clase tradicional para introducirse en situaciones más prácticas. Así, las aulas universitarias comenzarán a parecerse a los ambientes sociales y de trabajo del mundo real, que facilitan interacciones y la resolución de problemas interdisciplinarios (Johnson L. , 2016).

Dicho esto, a continuación, se analizarán dos líneas de acción: una basada en escenarios de aprendizaje y otra basada en actividades más que en contenidos.

\subsubsection{Método de escenarios de participación}

Este método se centra en la comprensión de los distintos modos de ser y de estar en la web. Para esto, es importante saber cómo los estudiantes utilizan la web y cómo esto incide en el uso de las interfaces digitales. La idea es partir de diferentes escenarios posibles de participación, como los siguientes: 
- Escenarios de inspiración (bibliografía y expertos)

- Escenarios de intercambio (foros, blogs, redes)

- Escenarios de creación colaborativa (actividades grupales)

- Escenarios de producción (e-portafolios)

- Escenarios de comunidad (participación en los diferentes espacios)

En este sentido, la propuesta abarca diferentes formas de acceder a la web con diferentes posibilidades de participación y exigencias.

\subsubsection{Aprendizaje centrado en actividades}

Esta metodología favorece el aprendizaje centrado en actividades más que en contenidos, lo que implica cambios profundos en el actuar del estudiante y del docente, así como en la forma de planificación del currículum, y del diseño y planificación de las asignaturas y sus respectivas clases. La Prof. B. Gros establece diferencias entre un proceso de enseñanza centrado en contenidos y uno que destaca las actividades. Este último sitúa al estudiante en el núcleo del proceso formativo, dado que favorece el aprendizaje colaborativo y autónomo (Gros, 2011). Este modelo se basa en la convicción de que los estudiantes aprenden mediante la realización y la interacción. Por ello, se fomentan actividades que privilegien el trabajo práctico, en equipo y relacionado con situaciones concretas, orientadas a la resolución de problemas o el desarrollo de habilidades de orden superior (Silva, 2017). A la par, mediante las e-actividades, los estudiantes emplean habilidades y competencias de diversa índole que, bajo metodologías activas apoyadas por las TIC, posibilitan un aprendizaje significativo, tanto a nivel individual como grupal.

\subsection{Acciones concretas: configuración de un curso virtual}

Al comenzar con el armado de un curso virtual, es esencial definir qué se desea elaborar, para qué y cómo. El diseño de las actividades que conforman el curso deben ser cuidadosamente seleccionadas, para que permitan lograr un alto nivel de implicación, motivación e interés hacia el curso, al tiempo que favorezcan un aprendizaje autónomo, activo y constructivo (Cabrero \& Roman, 2006). Para ello, es posible utilizar metodologías activas, como la búsqueda y procesamiento de la información, proyectos, estudio de casos, juegos de roles, la resolución de problemas, y el desarrollo colaborativo de productos y de debates. En cada actividad, se han de especificar las indicaciones específicas que deben ser ejecutadas para lograr el objetivo propuesto, el cual puede ser conceptual, actitudinal o procedimental.

Para cada clase, se debe crear una carpeta donde se ubiquen los materiales necesarios para la misma, los cuales se detallarán en la clase virtual (generalmente hipervinculados). En esta, será posible encontrar materiales didácticos, lecturas obligatorias y opcionales, sitios de Internet para visitar y materiales multimedia. Asimismo, se debe diseñar una serie de actividades, estableciendo foros de intercambio (técnicos, sociales o de trabajo) y la agenda de entrega de las tareas de la clase. 


\section{Cuadro 2. Diseño de clase (curso virtual)}

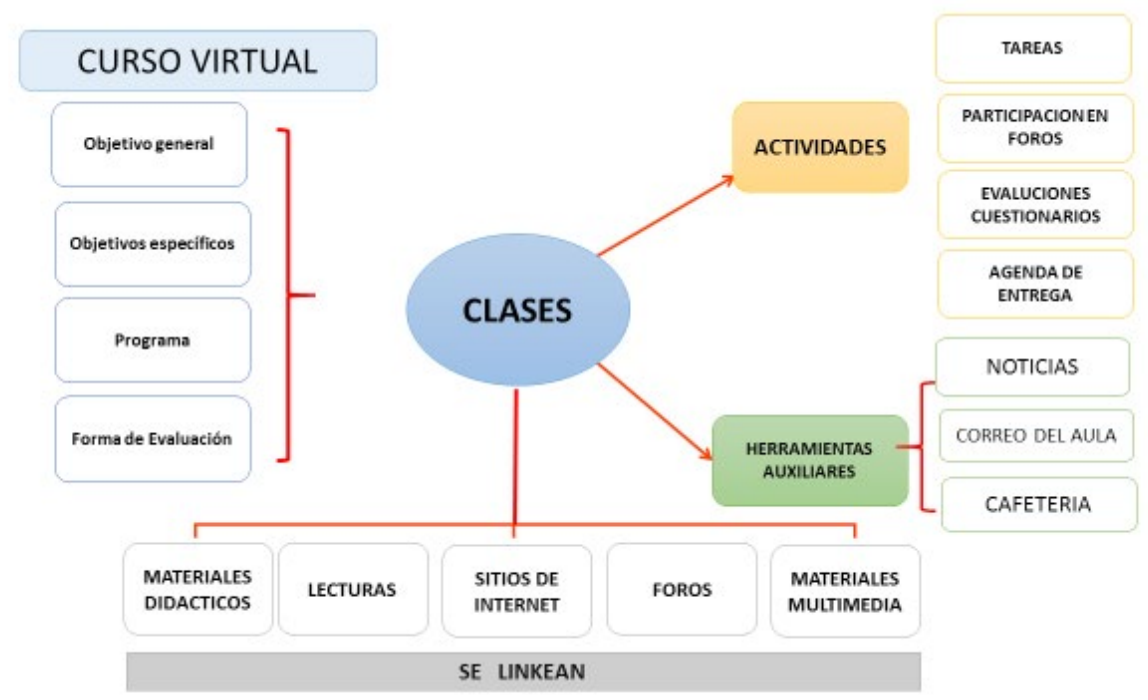

Fuente: Elaboración propia

Dentro del curso, siempre será necesaria un "Área de Herramientas auxiliares", donde se ubique un "servicio de noticias", que compende las novedades del curso, así como el "correo del aula", que se utilizará para la comunicación de docente a alumno, o entre alumnos. También, es interesante crear un "Área de Cafetería" para comunicaciones informales entre los participantes, que permita canalizar las emociones proyectadas por el estudiante al enfrentarse al aprendizaje. En lo que respecta a las actividades, pueden utilizarse, por un lado, actividades de trabajo colaborativo, como wikis, blogs o mapas conceptuales. Por otra parte, pueden diseñarse actividades que sirvan de autoevaluación de conocimientos, como distintos tipos de cuestionarios (múltiple opción, falso/verdadero, preguntas abiertas) o juegos evaluativos (crucigramas, correspondencias, simuladores) (Svetlichich, 2013).

\subsubsection{Características de los materiales}

Reconocer la existencia de diferentes estilos de aprendizaje permite presentar los materiales de diferentes formas para lograr que resulte fácil su comprensión para todos los que participen del curso (O'Connor \& Seymur, 1996). Este conocimiento permite al docente el desarrollo de diversas actividades que contemplen cómo aprende cada estudiante participante del curso (Svetlichich, 2013).

Se pueden distinguir cuatro estilos de aprendizaje con sus respectivas características:

a. Asimilador: el estudiante aprende en contacto con la información. Necesita leer, así como tomar apuntes prácticamente textuales.

b. Divergente: el estudiante debe saber porqué se actúa de un modo específico. Necesita estar motivado.

c. Convergente: el estudiante aprende mediante la práctica. No le interesa la teoría. 
Necesita conocer ejemplos.

d. Acomodador: el estudiante aprende a través del autodescubrimiento. Suele actuar de modo diferente a lo enseñado.

Al confeccionar el material educativo para un entorno virtual, es importante considerar que este debe ser de fácil lectura, emplear imágenes (fotos, dibujos o emoticones), videos, partes de películas u otros elementos que potenciarán la comunicación dentro del aula. Además, dado que se percibe a través de los sentidos y que cada persona posee una secuencia preferida en cuanto a su forma de percibir y procesar la información, es importante que los materiales utilizados se dirijan a los tres sentidos principales (vista, oído y tacto), a partir de la elección cuidadosa de las palabras para estimular el pensamiento en todos los canales sensoriales (Svetlichich, 2013).

Por último, es sustancial considerar los metaprogramas ${ }^{1}$ que están presentes en cada estudiante, los que permiten determinar "qué información le llega y cómo le llega" a cada uno de ellos (Svetlichich 2013). En este sentido, el estudiante está expuesto a mucha información y es su mente la que selecciona a qué prestar más atención. Frente a este contexto, cada metaprograma ofrece una variedad de posibilidades entre dos extremos, dado que la mayoría de las personas tiende, de manera natural, a operar más cerca de un extremo de posibilidad que del otro (Jago 2011), como se puede observar en el siguiente cuadro.

\section{Cuadro 3. Tipos de metaprogramas}
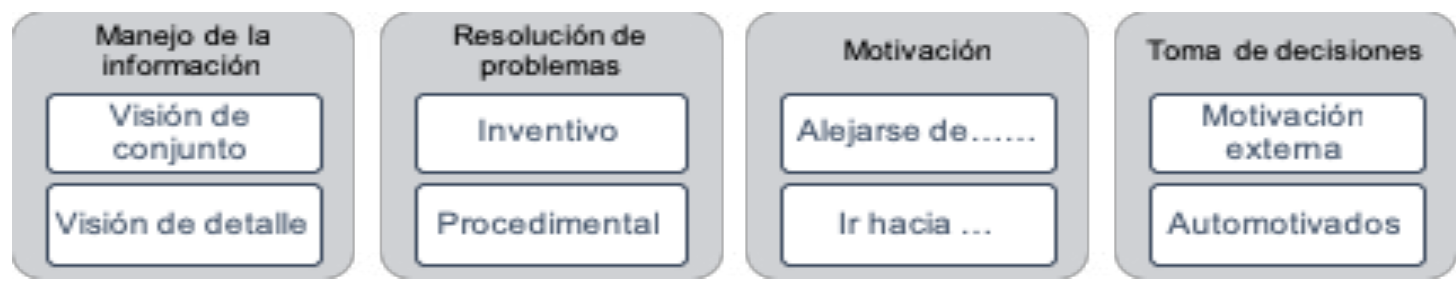

Fuente: Elaboración propia

\subsubsection{Los docentes participantes de la experiencia}

Dentro del modelo educativo actual, donde el estudiante juega un rol proactivo, el papel del docente no consiste solo en enseñar, sino también en propiciar que los alumnos aprendan (Dávila Espinoza, 2006). En esta línea, siguiendo a diferentes autores, se pueden definir cuatro roles básicos a desarrollar por los docentes. Estos se unifican ante la educación presencial y, muchas veces, se combinan por razones presupuestales ante proyectos virtuales. Son los siguientes:

- Docente académico: es el experto en el contenido de la materia.

- Docente contenidista: es el que realiza los materiales de la materia de acuerdo con

1 Estructuras mentales que operan un elevado nivel de generalidad para organizar una masa de información más específica. Actúan como plantillas o filtros que dejan pasar cierto tipo de información, mientras bloquean otra. Es un término técnico de Programación Neuro Lingüística (PNL) 
los contenidos fijados por el docente académico. Es un buen comunicador.

- Docente tutor en línea o docente asesor: es el que acompaña al alumno en su aprendizaje. Existen diferentes modelos de tutoría: por grupo de alumnos, por tema, por filtro de recepción de preguntas y traslado al experto, entre otros.

- Docente administrativo: es aquel que realiza las tareas de coordinación entre alumnos, tutores y docentes académicos.

Por último, es importante señalar que, en la educación virtual, si bien el contacto presencial entre docente y alumno es prácticamente inexistente, la comunicación es mucho más personalizada. De este modo, si bien el docente conoce a sus alumnos a distancia, muchas veces genera una relación más próxima y frecuente en el ámbito virtual, así como llega a conocer, y hasta anticipar, las necesidades y requerimientos de sus alumnos con mayor claridad que en una clase presencial, donde la masividad juega en contra de la relación interpersonal.

\section{Conclusiones}

El proyecto educativo debe ser asumido desde una perspectiva consecuente con la modalidad de formación seleccionada, requiriendo de la definición previa de los propósitos, contenidos, secuencia, método, recursos y tipos de evaluaciones que orientarán la labor académica de los docentes y estudiantes de un programa o curso académico. Además, los docentes deben estar dispuestos y comprometidos con la configuración de ambientes virtuales de aprendizaje significativo, que se sustentan en los lineamientos pedagógicos que garanticen la más alta calidad en los procesos de formación.

\section{REFERENCIAS BIBLIOGRÁFICAS}

Adell, J. (2004). Nuevas tecnologías en la formación presencial: del curso on line a las comunidades de aprendizaje. Qurriculum: Revista de teoría, investigación y práctica educativa, 57-92.

Bolivar, J., \& Rojas , F. (2014). Estudio de la autopercepción y los estilos de aprendizaje como factores asociados al rendimiento académico en estudiantes universitarios. Buenas Prácticas de Innovación Educativa - CINAIC .

Cabrero, J., \& Roman, P. (2006). Actividades Un referente basico para la formación en Internet. Sevilla: Eduforma.

Coll, C., Pozo, J., Sarabia, B., \& Valls, E. (1992). Los contenidos de la reforma: Enseñanaza y Aprendizaje de conceptos, procedimientos y actitudes. Madrid: Santillana.

Competencias del Siglo XXI. (20 de junio de 2017). Recuperado de http://www.fod. ac.cr/competencias21/index.php/acerca-de-las-competencias\#.WVPSG2g1_IV

Dávila, S. (2006). El aprendizaje significativo: esa extraña expresión utilizada por to- 
dos y comprendida por pocos. Contexto Educativo.

Diaz, F., \& Hernández, G. (2002). Estrategias docentes para un aprendizaje significativo. México: Mac Graw Hill.

Facer, K. (2011). Learning Futures. Education, technology and social change. Londres: Routledge.

Falco, M. (2017). Reconsiderando las prácticas educativas: TICs en el proceso de enseñanaza - aprendizaje. Tendencias pedagógicas $\mathrm{N}^{\circ} 29$.

García, L., Ruiz , M., \& Dominguez, D. (2007). De la educación a distancia a la educación virtual. Barcelona: Ariel.

Gonring, P; Ramsey, B. (2016). The William \& Flora Hewlett Foundation. Obtenido de http://www.hewlett.org/building-deeper-learning-field-grant/

Gros, B. (2011). Evolución y retos de la educación virtual: construyendo en el siglo XXI. BArcelona: UOC.

Higher, E. (2015). Everything you need to know about designing polysyncronous learning spaces. Insitute for the future. Recuperado de: http://www.iftf.org/home Jago, W. (2011). Transforma tu cerebro conPNL. Urano.

Jenkins, H. (2006). Convergence Culture: Where Old an New Media Collide. New YOrk University Press.

Johnson, L. (2016). NMC Informe Horizon. Texas: The New Media Consortium.

Johnson, S. (2005). Everything Bad is Good For You. How Today's Popular Culture is Actually Making US Smarte. New York: New York University Press.

Labrador, M., \& Andreu, M. (2008). Metodologías Activas. Valencia: Universisdad Politécnica de Valencia.

Llorente, M. (2005). La tutoría virtual: técnicas, herramientas y estrategias. Universidad de Sevilla.

Loprez, F. (s.f.). Globalización y Educación Superior en América Latina y el Caribe. IESALC/UNESCO.

O'Connor, J., \& Seymur, J. (1996). PNL Para Formadores. Urano.

OCDE. (2010). Inspired by Technology, Driven by Pedagogy: A Systemic Approach to Technology - Based School Innovations. En C. f. OCDE.

Peré, N. (2017). Apuntes para analizar la relación entre innovación, Tic y formación pedogógico didáctica. Praxis \& Saber, 15-33.

Rodes , V., \& Peré, N. (2005). Concepciones, modelos y prácticas de educación universitaria semipresencial y a distancia. Formación Docente, 61.

Rodriguez, M. (2012). El tutor como pieza clave en el rompecabezas de la educación en línea. Aprendizaje y mediación pedagogica con tecnologias digitales, (p. 352- 
361).

Silva, J. (2004). Diseño y moderación de entornos virtuales de aprendizaje.

Silva, J. (2017). Un modelo pedagógico vrtual centrado en las e-actividades. Revista de Educación a Distancia.

Svetlichich, M. E. (2011). Una mirada de la educación mediada con tecnología: la cercanía de la distancia. III Jornadas Académicas de la Facultad de Ciencias Económicas y Administración.

Svetlichich, M. (2013). Educación a distancia: Competencias docentes para alumnos interactivos. Uruguay: XXX CIC.

Svetlichich, M. (2015). La emoción de aprender: herramientas y técnicas de enseñanza - aprendizaje en un aula virtual. X Congreso de Educadores del Area Contable. Santo Domingo.

UNESCO. (2008). Estándares de Competencias TIC para docentes. Paris: UNESCO.

UNESCO. (2012). Aprendizaje movil para docentes en América Latina. Análisis del potencial de las tecnologías móviles para apoyar a los docentes y mejorar sus prácticas. Paris: UNESCO.

Urdaneta, M., \& Guanipa, P. (2007). Perfil de competnecias del docente tutor e línea pra la educación a distancia. Revista de Tecnología de Información y Comunicación en Educación, 111-134.

Wickline, H. (2014). The William \& Flora Hewlett Foundation. Obtenido de http:// www.hewllett.org/a-deeper-look-at-deeper-learning/

Recibido: 20/10/2018

Aceptado: 21/11/2018

Correspondencia: mariela.svetlichich@gmail.com 